See discussions, stats, and author profiles for this publication at: https://www.researchgate.net/publication/234443571

\title{
PLANCK-HFI : Performances of an optical concept for the cosmic microwave background anisotropies measurement
}

Article · May 2004

CITATIONS

4

8 authors, including:

Julien Brossard

French National Centre for Scientific Research

53 PUBLICATIONS 303 CITATIONS

SEE PROFILE

Yuying Longval

Institut d'Astrophysique Spatiale

59 PUBLICATIONS 1,013 CITATIONS

SEE PROFILE

Some of the authors of this publication are also working on these related projects:

LSPE - Large-Scale Polarization Explorer View project

PILOT balloon-borne experiment: measure of the dust emission View project
READS

22

V. B. Yurchenko

Gazi University

113 PUBLICATIONS 1,329 CITATIONS

SEE PROFILE 


\title{
PLANCK-HFI : performances of an optical concept for the cosmic microwave background anisotropies measurement.
}

\author{
J. Brossard ${ }^{(1,5)}$, V.Yurchenko ${ }^{(2)}$, E.Gleeson ${ }^{(2)}$, Y.Longval ${ }^{(3)}$, \\ B.Maffei ${ }^{(4)}$, A.Murphy ${ }^{(2)}$, I.Ristorcelli ${ }^{(5)}$, J-M.Lamarre ${ }^{(6)}$. \\ (1) CNES, Centre Spatial de Toulouse, 18, avenue Edouard Belin, 31401 Toulouse cedex4 - France. \\ Julien. Brossird(uicnes.fir. \\ (2) Dept. Of Experimental Physics, National University of Ireland, Maynooth, Co. Kildare, Ireland. \\ (3) Cardiff University. Dept. Of Physics and Astronomy. U.K. \\ (4) Institut d'Astrophysique Spatiale, Bât. 121, Université Paris Sud, 91495 Orsay Cedex, France.
}

(5) CESR, 9 Avenue du Colonel Roche, 31 401, Toulouse - France.

(6) LERMA, Observatoire de Paris, 61 Bd de l'Observatoire, 75014 Paris, France.

1. Abstract

PLANCK is a project of the European Space Agency to be launched in February 2007 by an ArianeV rocket with the Herschel Space Observatory . It is designed for imaging the temperature and polarization anisotropies of the millimetre and submillimetre radiation over the whole sky with unprecedented sensitivity, accuracy and angular resolution using 9 frequency channels ranging between 25 and $1000 \mathrm{GHz}$.

The main source at these frequencies is the Cosmic Microwave Background (CMB), i.e. the radiation emitted by the early universe when, about 300000 years old, ionised hydrogen recombined and became transparent from the visible to radio frequencies of the electromagnetic spectrum. The main goal of the PLANCK mission is to retrieve the main cosmological parameters of the Universe with accuracies of a few percent from the observation and analysis of random small contrast $\left(10^{-4}\right)$ features in the CMB. The angular power spectrum of the $\mathrm{CMB}$ anisotropies is a function of the fundamental cosmological parameters. A proper measurement of all the angular frequencies of the CMB is essential for an accurate interpretation of the data. In consequence the optical performances of Planck will directly impact the ability of retrieving theses parameters. Recent results of the Willkinson Microwave Anisotropy Probe (WMAP) mission show that polarization information of $\mathrm{CMB}$ radiation is very challenging, and that the precise measurement of the $\mathrm{CMB}$ could completely change the knowledge we have on our universe ([1]).

The focal plane assembly (FPA) of the PLANCK telescope is composed of two instruments. The High Frequency Instrument (HFI) of PLANCK is the most sensitive CMB experiment ever planned ([2]). Together with the Low Frequency Instrument (LFI), this will make a unique tool to measure the full sky and to separate various components of its spectrum.

This paper describes the main performances of the HFI beams and compares results obtained with 2 different softwares: GRASP8 [3] and an home-made software developed at the Ireland National University of Maynooth [4]. Specials attention will be paid to polarized beams $(100,143,217,353 \mathrm{GHz})$ and multimoded channels (545 and $857 \mathrm{GHz}$ ).

Keywords: ESA PLANCK Surveyor, CMB polarization, HFI Instrument, PO simulations, multimoded horns.

\section{Planck-HFI characteristics}

PLANCK telescope (see Figure 1) is a $1.5 \mathrm{~m}$ aperture dual off-axis antenna with 2 ellipsoidal mirrors [5]. The surface accuracy of the mirrors will be less than $10 \mu \mathrm{m}$ with a roughness less than $1 \mu \mathrm{m}$.

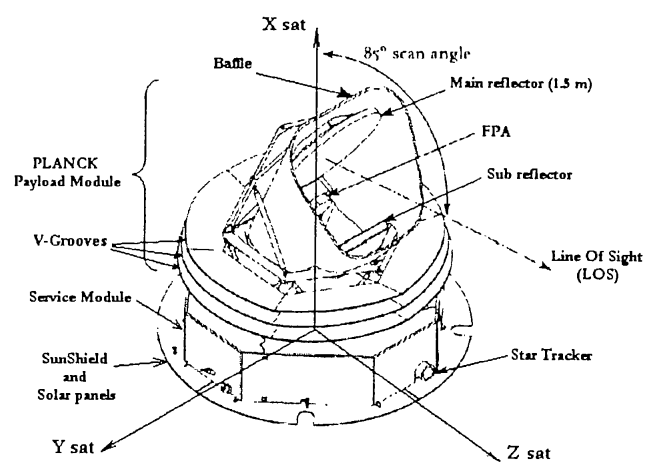

Figure 1 : PLANCK Satellite, and his associated coordinate system (Xsat,Ysat,Zsat).

The HFI consists of 36 corrugated homs feeding cryogenically cooled bolometric detectors. Each bolometer is coupled with the telescope using a 3horns configuration scheme presented in Figure 2. A corrugated back to back horn is facing the sub-reflector of the telescope, giving a low sidelobes level and an other horn (at $100 \mathrm{mK}$ ) is used to feed the power to a bolometer situated at the end of it. Filters and lenses located between on these stages provide most of the desired frequency selection. Cut-off of low frequencies is partly achieved in the waveguide sections of the horns. This configuration allows a control of the 
background and thermal environment from the back to back horn at $4.5 \mathrm{~K}$ to the bolometers at $0.1 \mathrm{~K}$ [6].

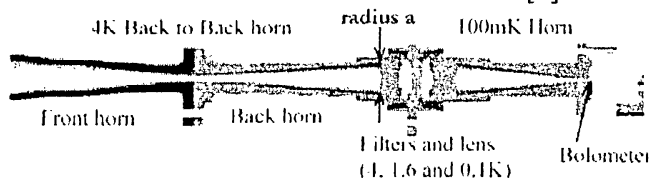

Figure 2 : PLANCK-HFI Horn scheme.

The position and the orientation of the horns are defined in the Reference Detector Plane (see Figure 3 and reference [7]). Channels operating at 100, 143, 217 and $353 \mathrm{GHz}$ use single-mode horns. Half of these channels are equipped with polarization-sensitive bolometers (PSB, noted (p) in Table 1). The two highest frequency channels (545 and $857 \mathrm{GHz}$ ) are unpolarized and incorporate multimoded horns. All bandwidths are about $30 \%$ of the central frequency. Table 1 shows the principal HFI optical characteristics, in term of angular resolution on the sky and edge taper on the primary mirror. Figure 6 shows the PLANCKHFI main beam position on the sky. The position of the horns in the FPA and the geometrical configuration of the mirrors leads to nearly aligned beams along lines on the sky parallel to the scanning direction $\left(\theta^{\mathrm{SC}}=\mathrm{cst}\right)$. This configuration has been chosen in order to minimize some systematic effects and to facilitate some aspects of the data processing.

The orientation of the beam polarization angle $\tau$ on the sky is given in Table 2 for the channel "a". The orientation of the complementary b-channel is orthogonal to the a-channel one in the FPA. The definition of the angle of polarization $\tau$ is presented in Table 4 ( $\vec{n}$ unit vector is along the major axis of the ellipse described by the electrical field along the maximum power of the beam).

\begin{tabular}{|c|c|c|c|}
\hline Band & $\begin{array}{c}\text { Number of } \\
\text { channels }\end{array}$ & $\begin{array}{c}\text { Angular } \\
\text { resolution } \\
\text { (arcmin) }\end{array}$ & Edge Taper \\
\hline $100 \mathrm{GHz}$ (mono) & $4(\mathrm{p})$ & 9.5 & $-27 \mathrm{~dB}$ \\
\hline $143 \mathrm{GHz}$ (mono) & $4+4(\mathrm{p})$ & 7.1 & $-28 \mathrm{~dB}$ \\
\hline $217 \mathrm{GHz}$ (mono) & $4+4(\mathrm{p})$ & 4.7 & $-30 \mathrm{~dB}$ \\
\hline $353 \mathrm{GHz}$ (mono) & $4+4(\mathrm{p})$ & 5 & $-40 \mathrm{~dB}$ \\
\hline $545 \mathrm{GHz}$ (multi) & 4 & 5 & $-29 \mathrm{~dB}$ \\
\hline $857 \mathrm{GHz}$ (multi) & 4 & 5 & $-29 \mathrm{~dB}$ \\
\hline
\end{tabular}

Table 1 : HFI optical requirements (mono : monomode, multi : multimoded)

\begin{tabular}{|c|c|}
\hline Band & $\tau$ angle for channel-a \\
\hline $100-1$ & $+22.5^{\circ}$ \\
\hline $100-2$ & $+45^{\circ}$ \\
\hline $100-3$ & $0^{\circ}$ \\
\hline $100-4$ & $-22.5^{\circ}$ \\
\hline $143-1$ & $+45^{\circ}$ \\
\hline $143-2$ & $+45^{\circ}$ \\
\hline
\end{tabular}

\begin{tabular}{|c|c|}
\hline $143-3$ & $0^{\circ}$ \\
\hline $143-4$ & $0^{\circ}$ \\
\hline $217-5$ & $+45^{\circ}$ \\
\hline $217-6$ & $+45^{\circ}$ \\
\hline $217-7$ & $0^{\circ}$ \\
\hline $217-8$ & $0^{\circ}$ \\
\hline $353-3$ & $+45^{\circ}$ \\
\hline $353-4$ & $+45^{\circ}$ \\
\hline $353-5$ & $0^{\circ}$ \\
\hline $353-6$ & $0^{\circ}$ \\
\hline
\end{tabular}

Table 2 : Definition of the angle of polarization on the sky for all a-polarized channels.

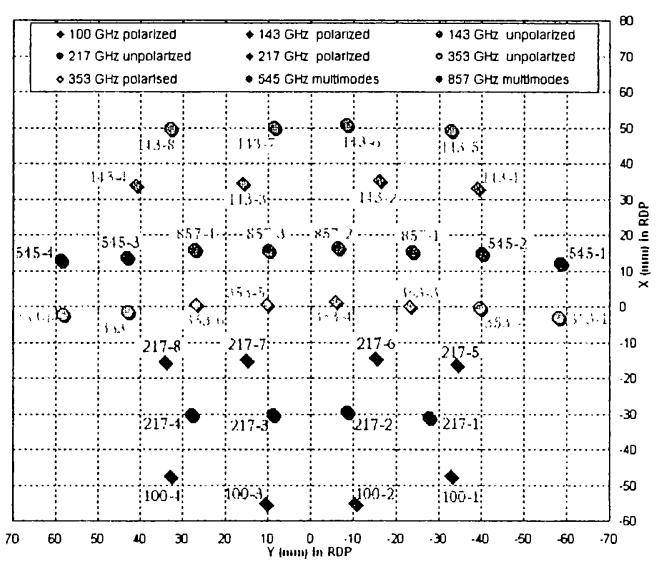

Figure 3 : Projection of the phase centre of HFI horns onto the $(\mathrm{X}, \mathrm{Y}) \mathrm{RDP}$ plane.

1. Is the full radiation pattern of the PLANCK-HFI accessible?

The Scientific goals of the mission impose unprecented requirements onto the optical properties of PLANCK satellite. Very low side lobes are needed to control straylight. In addition, the full radiation pattern should be very accurately know because the inversion of the scientific data critically depends on its exact real shape. It has not been proved that measurements on the ground or in flight could provide a good enough information. This particularly the case for the polarization properties of the experiment, since no polarization standard is currently known in the sub $\mathrm{mm}$ sky.

However, the precise and complete $(4 \pi)$ characterisation of the radiation pattern is extremely difficult:

- by computation, due to the ratio of the mirrors to the wavelength (up to 5000) and to the mumber of elements. The very low levels required may also hit the limits of the inexact representation of a real (imperfect) device. 
- by measurement due to the high contrast between the main beam and the far side lobes and to the presence of the absorbing atmosphere for some of the bands. In addition, compact range facilities are not often qualified for sub-terahertz frequencies.

Dual reflector main beams antenna modelling is rather usual, and the Physical optics (PO) technique is the most suitable for such simulations. In the case of PLANCK, the computation is complicated because of the large electrical size of the main reflector $\left(D / \lambda_{\min } \sim 5000\right)$ and the multimoded propagation for the higher frequency channels [8]. Polarization properties of the telescope should be analysed and characterized with a high precision [9].

Concerning the simulation of the far side lobes, the whole geometry of the spacecraft has to be considered (baffle, sun-shield, V-grooves). The Geometrical Theory of Diffraction (GTD) is the most suitable technique for such a purpose. For PLANCK-HFI, some preliminary analysis has already been done using approximation to the real far-field of the horns [10]. For a precise side-lobe evaluation, the real field feed pattern has to be considered into the simulation.

On ground measurement of the Planck satellite is confronted to others difficulties. At the present time, the measurement of large antenna $(\varnothing=1.5 \mathrm{~m})$ begins to be accessible at $500 \mathrm{GHz}$ for few degrees around the main beam only [11]. Antenna Test Range (Compact range, Holographic range) are significantly analysed and optimised for the millimetre and sub-millimetre band $[12,13,14]$. But still today, the level of precision required for a full-frequency in $4 \pi$ steradians analysis of Planck is not yet accessible.

As a preliminary conclusion, it should be noted that the PLANCK-HFI radiation pattern requirement imposes a comparison between computation and measurement results. Both aspects (theoretical and experimental) have to be fully used on the ground and in flight.

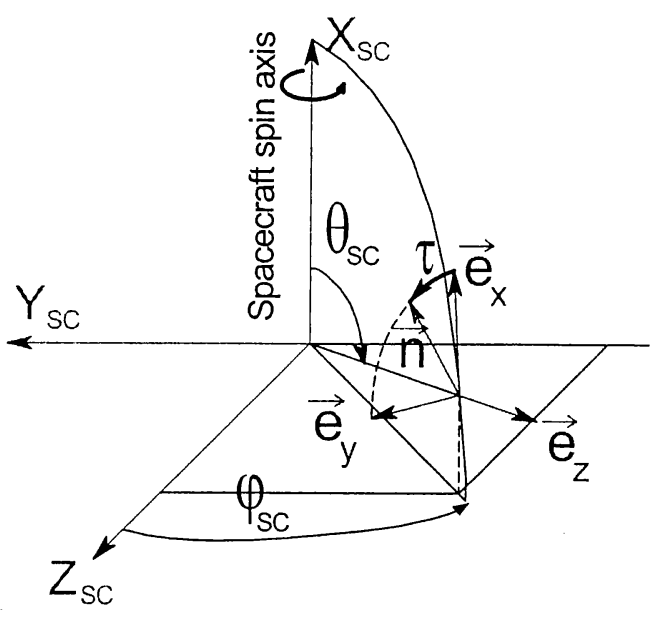

Figure 4 : Angles $\left(\theta^{\mathrm{SC}}, \phi^{\mathrm{SC}}\right)$ used for the projection of the main beams on the sky. The Spacecraft coordinate system being defined in ref [5]. The Cartesian frame

$\left(\vec{e}_{x}, \vec{e}_{y}, \vec{e}_{z}\right)$ is used to define the Stokes parameters $\mathrm{Q}$ and $\mathrm{U}$.

\section{Main beams computation for monomode} horns:

2.1: Unpolarized horns:

There are 12 unpolarized detectors (see Figure 3). The horn radiation depends on the frequency and the internal geometry of the horn. The internal geometry was optimised by Cardiff and the Experimental department of Physics of the National University of Ireland (NUI) using the commercial software "CORRUG" and a home-made software specially developed for PLANCK application. Figure 5 shows the far-field of the horn $100-1$ at $100 \mathrm{GHz}$ given after this optimisation. The optimisation process has been obtained after a trade-off between the angular resolution and the level of spillover (related to the edge taper of Table 1) of the telescope far-field [15].

The telescope main beam is computed by propagating the horn field into telescope through the far-field. Different approximations can be used for such computation. At $100 \mathrm{GHz}$, the main beam is obtained with Physical Optics (PO) on both reflectors. At higher frequencies (143, 217 and 353) a complete (sub+main) PO analysis is very time consuming and an other method is used (excepted for the NUI home-made software [9]). This method, using GO and PO analysis is valid because the sub-reflector is in the far field of the horn. In this hybrid method, the propagation onto the sub reflector is performed with the GO, and the Physical Optics is used to compute the currents at the surface of the main reflector. The telescope far-field 
being obtained by summing all the elementary fields radiated by these individual currents [3].

Cross-correlation, between GRASP8 and NUI home-made software shows that the results are very similar. The NUI-PLANCK software being faster than GRASP8, but - at present time - limited to the main beam computation.

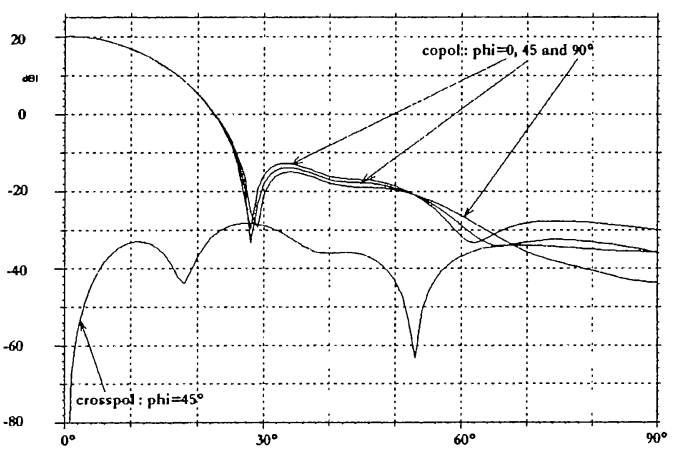

Figure $5: 100 \mathrm{GHz}$ horn far-field for the horn number 100-1.

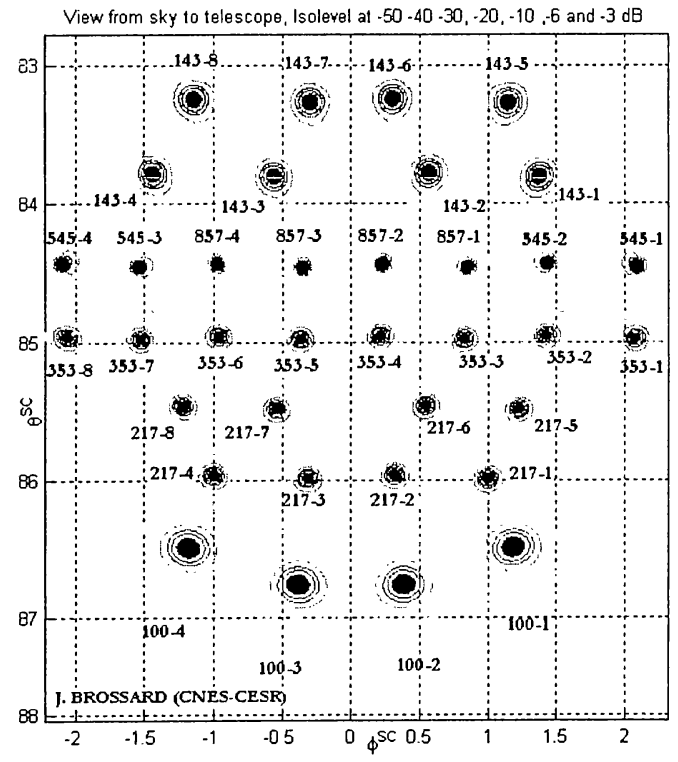

Figure $6:$ Normalized power main beams of PLANCK-HFI view from sky to telescope and projected onto the Spacecraft frame defined in Figure 4. The red part corresponds to levels higher than $-3 \mathrm{~dB}$. The others contours are $-6,-10,-20,-30,-40$ and -50 $\mathrm{dB}$ below the power-barycentre of the beam. The angles $\theta^{\mathrm{SC}}$ and $\phi^{\mathrm{SC}}$ are in degrees.
2.2: Polarized horns :

The polarization properties of the PLANCK telescope should be known with a high accuracy, because the level of polarization anisotropies of the $\mathrm{CMB}$ is expected to be less than $10 \%$ of the temperature fluctuations.

The polarized horns are equipped with Polarized Sensitive Bolometer (PSB) giving the electrical field amplitudes along 2 orthogonal axis in the FPA. Each polarized channel (one PSB giving 2 channels) has its own power main beam on the sky. The 2 channels of the same polarized horn of PLANCK have nearly the same power main beam on the sky, with small differences $(\sim 1 \mathrm{~dB})$ at low level $(-20 \mathrm{~dB}$ below the maximum) (see Figure 7, where beams have been computed using PO on both reflectors).

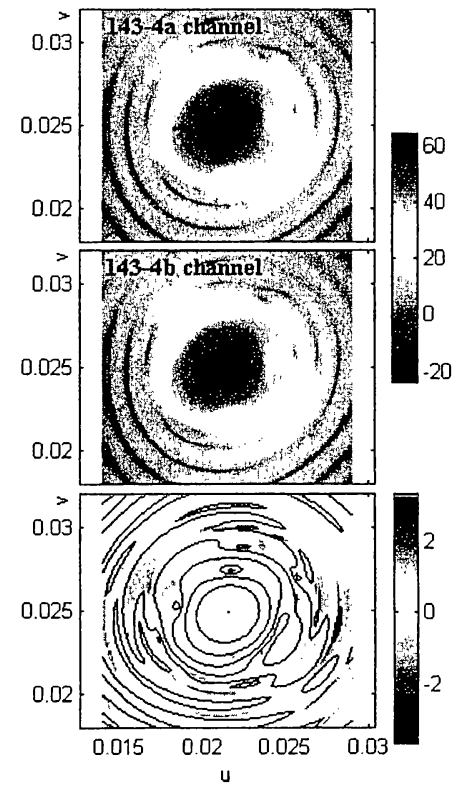

Figure 7 : Power main beam of the channel-a and channel-b of the horn 1434 and the difference (third figure). On the last figure the iso- $\mathrm{dB}$ level are at -60 , $50,-40,-30,-20$ and $-10 \mathrm{~dB}$ below the maximum of power. The beams are projected onto $\left(\mathrm{u}^{\mathrm{LOS}}, \mathrm{v}^{\mathrm{LOS}}\right)^{1}$ frame.

The polarization characteristics of the polarized beams can be estimated using the Stokes parameters defined by :

$$
\begin{aligned}
& I=\left\langle\left. E_{x}\right|^{2}\right\rangle+\left\langle\left. E_{y}\right|^{2}\right\rangle \\
& Q=\left\langle\left. E_{x}\right|^{2}\right\rangle-\left\langle\left. E_{y}\right|^{2}\right\rangle
\end{aligned}
$$

$1 \mathrm{u}^{\mathrm{LOS}}=\sin \theta^{\mathrm{LOS} *} \cos \phi^{\mathrm{LOS}}, \mathrm{v}^{\mathrm{LOS}}=\sin \theta^{\mathrm{LOS} *} \sin \phi^{\mathrm{LOS}}$. 


$$
\begin{aligned}
& U=\left\langle E_{x} E_{y}{ }^{*}\right\rangle+\left\langle E_{y} E_{x}^{*}\right\rangle \\
& V=i\left(\left\langle E_{x} E_{y}{ }^{*}\right\rangle-\left\langle E_{y} E_{x}{ }^{*}\right\rangle\right)
\end{aligned}
$$

Where $E_{x}$ and $E_{y}$ are the complex amplitudes of the electric field along the $\mathrm{x}$ and $\mathrm{y}$ axis of the Cartesian coordinate system used as reference. In the case of PLANCK, the $\mathrm{V}$ parameter of the CMB radiation is supposed to be zero (no circular polarization), so no characterisation of this parameter is required. $Q$ and $U$ parameters are defined in a frame-dependent way (the I Stokes parameter being the total power of the wave is not frame dependent). Hence, the polarization property of the main beam, using Q and U Stokes parameters, has to be referenced to a well defined Cartesian coordinate system. In this paper, the $Q$ and $U$ parameters are defined in the $\left(\vec{e}_{x}, \vec{e}_{y}, \vec{e}_{z}\right)$ frame defined as follow (see Figure 4):

$$
\begin{aligned}
& \vec{e}_{x}=\sin \theta^{S C} \vec{e}_{X^{s c}}+\cos \theta^{S C} \sin \varphi^{S C} \vec{e}_{Y^{s c}}-\cos \theta^{s C} \cos \varphi^{S C} \vec{e}_{Z^{s c}} \\
& \vec{e}_{y}=\cos \varphi^{S C} \vec{e}_{Y^{\prime S C}}+\sin \varphi^{S C} \vec{e}_{Z^{s c}} \\
& \vec{e}_{z}=\cos \theta^{S C} \vec{e}_{X^{s c}}-\sin \theta^{S C} \sin \varphi^{S C} \vec{e}_{Y^{\prime s C}}+\sin \theta^{S C} \cos \varphi^{S C} \vec{e}_{Z^{s c}}
\end{aligned}
$$

Figure 8 and Figure 9 (resp. Figure 10 and Figure 11) shows the $U$ (resp. V) Stokes parameter map for the horn 143-4a of HFI and 2 different computations, projected onto the $\left(\eta_{\mathrm{SC}}=\pi / 2-\theta_{\mathrm{SC}}, \varphi_{\mathrm{SC}}\right)$ frame. In case of NUI computation, the map is an average of the Stokes parameter inside the full bandwidth. For Grasp8 computation, the map is mono-frequency (at the center of the band). Despite this difference, the results are very similar.

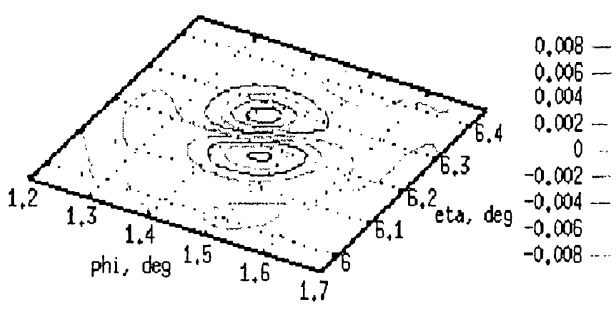

Figure 8 : U stokes parameter of the beam at 143 $\mathrm{GHz}$ for the horn 143-4a. (NUI result, phi=-phi $i_{S C}$ ). Figure from [9].

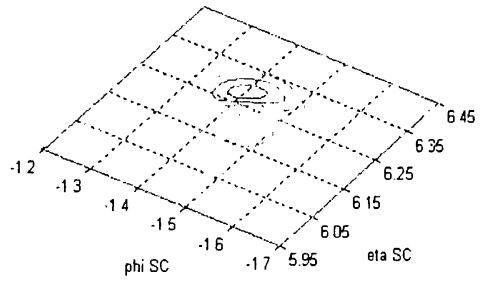

Figure 9 : U stokes parameter of the beam at $143 \mathrm{GHz}$ for the horn 143-4a. (GRASP8 result). The isolevels are the same as in Figure 8.

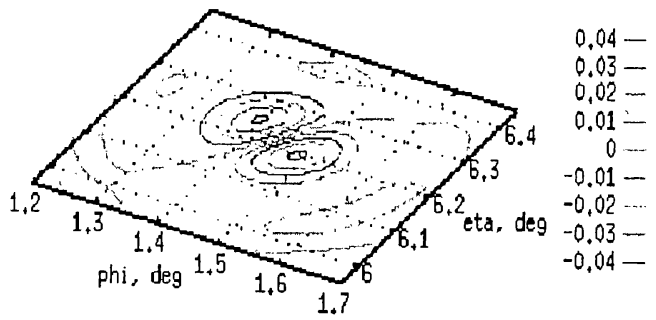

Figure $10: \mathrm{V}$ stokes parameter of the beam at 143 $\mathrm{GHz}$ for the horn 143-4a (NUI result, phi=-phi $\mathrm{SC}_{\mathrm{SC}}$ ). Figure from [9].

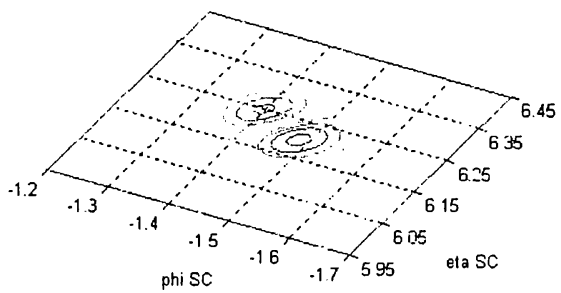

Figure $11: \mathrm{V}$ stokes parameter of the beam at 143 $\mathrm{GHz}$ for the horn 143-4a (GRASP8 result). The isolevels are the same as in Figure 10.

3. Main beam computation for multimoded horns :

The horn far-field is computed using the scattering matrix method [13]. In this method, the starting point of the modelling process is the mouth of the back-toback horn facing the $100 \mathrm{mK}$ horn (shows with 2 arrows in Figure 2). The horn corrugation are regarded as a collection of waveguide sections of varying radii and so modelled as a sequence of discontinuities. Modes allowed to propagate into a uniform waveguide of radius "a" (see Figure 2) are launched at the back of the horn (these modes are the usual TE and TM modes allowed to propagate into smooth circular infinite waveguide). Then propagation inside the horn is performed using Maxwell equations. For one exciting mode at the back of the hom, we obtain a complex summation of TE and TM modes at the aperture horn facing the sub reflector of the telescope. The far-field of the horn (for the excited mode under consideration) is computed using a simple Fourier transform of the aperture field. This far field is then propagated into the telescope. This procedure is repeated for all the modes leading to non-negligible power on the sky and the total power is obtained by summing all the individual 
power on the sky. Here, we supposed that all the modes at the back of the back to back horn carry the same power [14]

The number of excited modes used for the computation presented in this paper is 5 (resp. 9) at 545 $\mathrm{GHz}$ (resp. $857 \mathrm{GHz}$ ). Table 3 shows the excited modes needed for an accurate HFI-PLANCK telescope far-field computation.

\begin{tabular}{|c|c|c|}
\hline & $545 \mathrm{GHz}$ & $857 \mathrm{GHz}$ \\
\hline $\begin{array}{c}\text { Excited } \\
\text { modes }\end{array}$ & $\mathrm{TE}_{01}, \mathrm{TM}_{01}, \mathrm{TE}_{11}$, & $\mathrm{TE}_{01}, \mathrm{TM}_{01}, \mathrm{TE}_{12}$, \\
& $\mathrm{TE}_{21}, \mathrm{TM}_{02}$. & $\mathrm{TE}_{11}, \mathrm{TE}_{21}$, \\
$\mathrm{TM}_{21}, \mathrm{TE}_{41}, \mathrm{TE}_{31}$. \\
\hline
\end{tabular}

Table 3 : Excited modes used for the HFI-telescope farfield computation at 545 and $857 \mathrm{GHz}$.

Figure 12 shows the construction - at $857 \mathrm{GHz}$ - of the Planck power main beam for the horn "857-1", function of the number of excited mode used in the computation. We can see that the number of modes necessary for an accurate modelling is 9 (exactly the modes listed in Table 3). Figure 13 shows the individual modes contribution to the total power of the beam, for a spherical cut passing through the barycentre of the beam.

Another effect has to be considered if we want to have a correct prediction of the power beam. As said previously, the radiation pattern of a horn is frequency dependent. Then, inside the HFI-band width, the beam shape will vary. This is true even for monomode horns, but for multimode horns it is more complex because, each individual mode has a different frequency dependency. It has also to be noticed that the selection of the modes is also frequency dependent (for example, the mode TE41 used in the modelling at $857 \mathrm{GHz}$ is not taken into account at $716 \mathrm{GHz}$ ).

For those reasons, a precise modelling of the telescope main beam inside the full bandwidth has been performed. Table 4 shows the power main beam width variation (for the horn $n^{\circ} 857-1$ ) with respect to 5 equi-spaced mono-frequency values in the band. We can see that the average full-width half maximum (FWHM) is near the specification (see Table 1). The beam width at $-3 \mathrm{~dB}$ is varying from $4,3^{\prime}$ to $5,7^{\prime}$ inside the band. The relative maximum variation of the FWHM - with respect to the central frequency - reach $17 \%$ (at $716 \mathrm{GHz}$ ) and the specification is not strictly respected in the very low frequencies part of the band.

For a rigorous modelling, a polarization orientation average of the feed has to be added [9], but it is supposed to be small.

As a conclusion, the fine modelling of the multimoded main beam of PLANCK-HFI should take into account different sources of systematic:

- broad band effect $\begin{array}{ll}\text { - } & \text { modes effect } \\ \text { - } & \text { polarization effect }\end{array}$

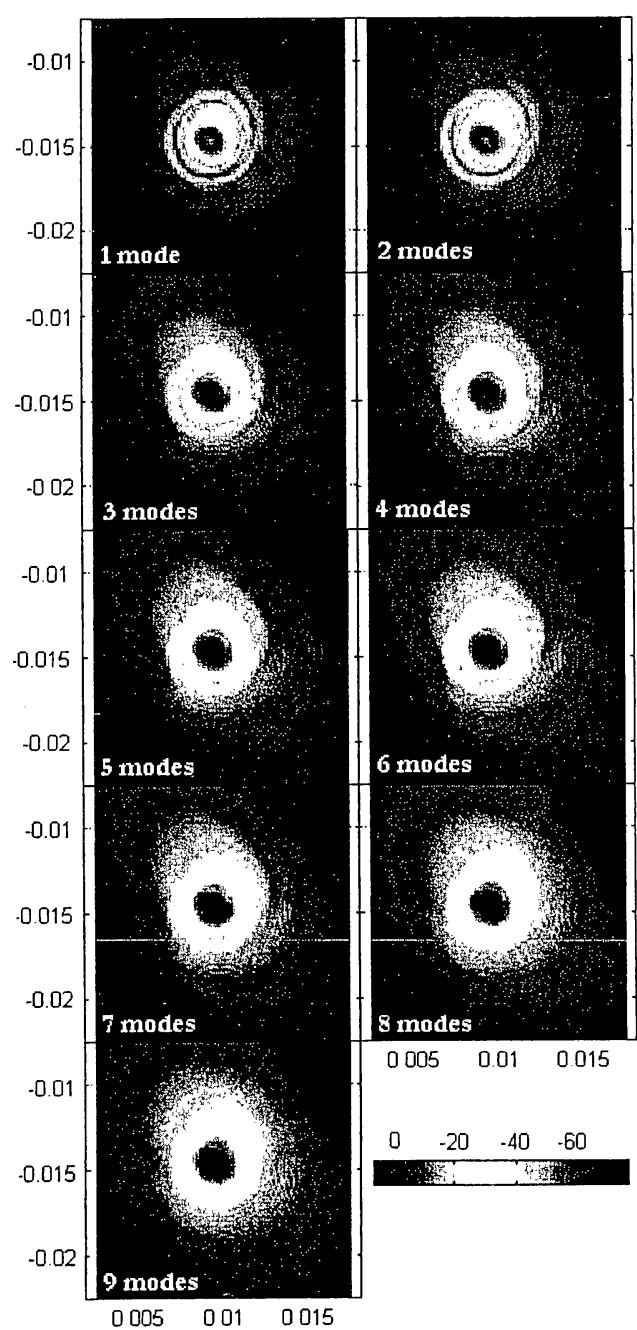

Figure 12 : Planck-HFI beam construction for the horn $\mathrm{n}^{\circ} 857-1$. The beam is projected onto the $\left(\mathrm{u}^{\mathrm{LOS}}, \mathrm{v}^{\mathrm{LOS}}\right)^{2}$ coordinate system of the "Line Of Sight" frame ([11]).

${ }^{2}: \mathrm{u}^{\mathrm{LOS}}=\sin \theta^{\mathrm{LOS} *} \cos \phi^{\mathrm{LOS}}, \mathrm{v}^{\mathrm{LOS}}=\sin \theta^{\mathrm{LOS} *} \sin \phi^{\mathrm{LOS}}$ 


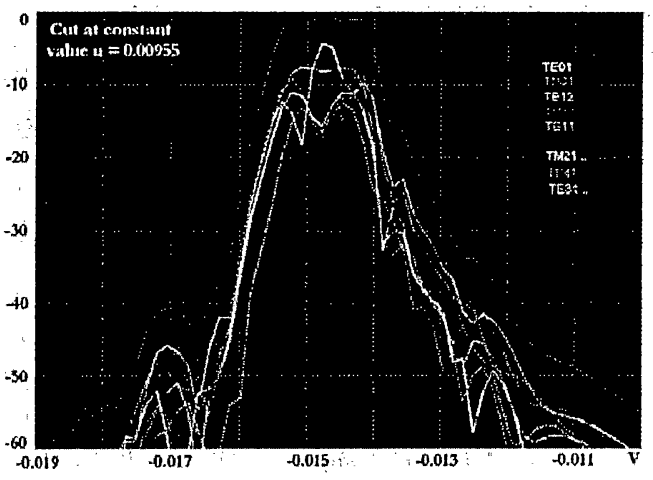

Figure 13: Cut at constant $u^{\text {LOS }}$ value $\left(u^{\operatorname{LOS}}=0.00955\right)$ of the power pattern for the horn $857-1$ at $857 \mathrm{GHz}$, passing through the barycentre of the beam main. The individual mode contribution is shown in color. The brown curve being the summation of the individual power modes.

\begin{tabular}{|c|c|c|c|}
\hline & $\begin{array}{c}\text { BW at } \\
-3 \mathrm{~dB}\end{array}$ & $\begin{array}{c}\text { BW at } \\
-6 \mathrm{~dB}\end{array}$ & $\begin{array}{c}\text { BW at } \\
-10 \mathrm{~dB}\end{array}$ \\
\hline $716 \mathrm{GHz}$ & $5.5^{\prime}$ & $6.5^{\prime}$ & $7.6^{\prime}$ \\
\hline $786 \mathrm{GHz}$ & $5.0^{\prime}$ & $5.9^{\prime}$ & $6.7^{\prime}$ \\
\hline $857 \mathrm{GHz}$ & $4.7^{\prime}$ & $5.4^{\prime}$ & $6.1^{\prime}$ \\
\hline $927 \mathrm{GHz}$ & $4.6^{\prime}$ & $5.3^{\prime}$ & $5.9^{\prime}$ \\
\hline $998 \mathrm{GHz}$ & $4.3^{\prime}$ & $4.9^{\prime}$ & $5.3^{\prime}$ \\
\hline Average & $4.8^{\prime}$ & $5.6^{\prime}$ & $6.2^{\prime}$ \\
\hline
\end{tabular}

Table 4 : Beam width (BW) at different levels for 5 frequencies in the $857 \mathrm{GHz}$ band of HFI (horn 857-1).

\section{Conclusion :}

In this paper, we present some of the major "optics" characteristics of the main beams of planckHFI. The optimal design gives angular resolutions and beam lines of sight in agreement with the requirements. The polarized and multimoded main beams modeled with GRASP8 and NUI home-made software show very similar results, giving confidence in the modelling.

We have shown the very complex process implied in the precise modelling of the high frequencies channels of PLANCK-HFI (multimoded channels).

We have now a better knowledge of the main beams properties of the HFI-PLANCK satellite thanks to two different electromagnetic modellings (Grasp8 and NUI home-made software). The experimental characterisation of some main beams and part of the far side lobes should normally be performed in 2005 during the Radio-Frequency Qualification Model measurement.

Concerning the far-side lobes, there is still a lot of work to do (both from modelling and experimental aspects), in order to completely control and characterize the Satellite far-field pattern.

\section{References :}

[1] : "Dodecahedral space topology as an explanation for weak wide-angle temperature correlations in the cosmic microwave background", J-P. Luminet \& al., Nature 425, 593-595 (2003).

[2] : "The Planck High Frequency Instrument, a $3^{\text {rd }}$ generation $\mathrm{CMB}$ experiment, and a full sky submillimeter survey", J-M. Lamarre \& al., Proceedings of the workshop on "The Cosmic Microwave Background and its polarization", New Astronomy reviews, (astro-ph/0308075).

[3] : "Technical Description of GRASP8", K. Pontoppidan, edited by TICRA Engineering Consultants, Copenhagen, Denmark, February 2003.

[4] : "Fast-optical Optics Simulations of the Dualreflector Submillimeter-Wave Telescope on the ESA Planck Surveyvor", V. Yurchenko and J.A.Murphy, $8^{\text {th }}$ International Conference on Terahertz electronics, 153-156, Darmstadt, Germany, September 2000

[5] : "PLANCK Telescope Design Specification", ESA Document, ref. SCI-PT-RS-07024, Issue 1, revision: 0, 31 August 2000.

[6] : "Planck-HFI Optical Concept", B.Maffei \& al., Proceeding of the $28^{\text {th }}$ International Conference on Infrared and Millimeter Waves, 2003.

[7] : "Position and Orientation of HFI Horns", Y.Longval, HFI document, ref. TN-PH234100098-IAS, 24 Jan. 2003, Issue : 1, revision 3.

[8]: "Simulations of the submillimeter-wave telescope on the ESA PLANCK surveyvor", V.B. Yurchenko, MSMW'2001 symposium Proc., Kharkov, June 4-9, 2001, pp. 791-793.

[9] : "Simulation and comparison of the PLANCK HFI beams with implications on polarization measurements", V.B. Yurchenko, Proc. $3^{\text {rd }}$ ESA Workshop on Millimeter Wave Technology and Applications, 21-23 May, 2003, Millilab, Espoo, Finland, pp. 187-192 (2003).

[10]: "Planck RF expertise, Phase B, WP2, Far out side lobe radiation pattern computation", P.H. Nielsen, TICRA engineering consultants document, S-1117-05, November 2002.

[11]: "Calibration and verification measurement in compensated compact ranges up to $500 \mathrm{GHz}$ ", J. Hartmann \& al., Proceeding of the $23^{\text {rd }}$ "Antenna Measurement Techniques Association", 2001.

[12] : "Design and construction of a $200 \mathrm{GHz}$ Demonstrator of a Tri-Reflector Compact Antenna Test Range with spherical Main reflector", Parini \& al., $22^{\text {nd }}$ ESTEC Antenna Workshop on Antenna Measurement", 1999. 
[13] : "Dual-Reflector compact range modelling : geometrical theory of diffraction analysis and sub-millimeter quiet-zone computation",

J.Brossard \& al., Journal of Optics : pure and applied optics, 5 (2003), 108-116.

[14] : "Development of a $310 \mathrm{GHz}$ hologram Compact antenna test range", Antenna Measurement Techniques Association Proceedings, 1999, Monterey Bay, California.

[15] : "Radiation patterns of multi-moded corrugated horns for far-IR space applications", J.A.

Murphy \& al., Infrared Physics and Technology, Vol. 42, No 6, December 2001.

[16]: "Definition of the Multi-moded Horns for the HFI Instrument on PLANCK", E. Gleeson \& al., $25^{\text {th }}$ Antenna Workshop on Satellite Antenna Technology. 